\title{
Financial impact of health care-associated infections: When money talks
}

\author{
Louis Valiquette MD MSc FRCPC ${ }^{1}$, Claire Nour Abou Chakra MSc ${ }^{1}$, Kevin B Laupland MD MSc FRCPC ${ }^{2,3}$
}

A ccording to the Canadian Institute for Health Information, health spending in Canada was projected to reach $\$ 211$ billion in 2013 (versus $\$ 207$ billion in 2012), corresponding to $\$ 5,988$ per person (1). Overall, this represents $11.2 \%$ of Canada's gross domestic product. Approximately $60 \%$ of total health spending is directed to hospitals $(30 \%)$, drugs $(16 \%)$ and physicians (15\%). Although it is difficult to estimate, the proportion of this spending attributed to the management of nosocomial infections, overuse and/or misuse of antimicrobials, and infections due to multidrug-resistant bacteria is significant. Despite the availability of efficient strategies targeting each of these aspects, large-scale progress has not been demonstrated.

In a recent meta-analysis, Zimlichman et al (2) included 26 studies and used incidence estimates from the National Healthcare Safety Network of the Centers for Disease Control and Prevention, formerly known as the National Nosocomial Infections Surveillance (NNIS). They estimated the costs and excess length of stay (xLOS) associated with significant health care-associated infections (HAIs). On a per-case basis, central lineassociated bloodstream infections (CLABSIs) were found to be the most costly (US $\$ 45,814$ [2012]; xLOS 10.4 days), followed by ventilatorassociated pneumonia (US\$40,144; xLOS 13.1 days) and surgical site infections (US $\$ 20,785$; xLOS 11.2 days). When caused by methicillinresistant Staphylococcus aureus (MRSA), both the cost and xLOS of surgical site infections increased by $105 \%$; CLABSI cost increased by $22 \%$ and CLABSI xLOS increased by $51 \%$. These results highlight the importance of strategies to control bacterial antibiotic resistance.

The three most significant antibiotic-resistant bacteria found in Canadian centres are MRSA, vancomycin-resistant enterococci (VRE) and extended-spectrum beta-lactamase (ESBL)-producing organisms. A summary of a literature review is shown in Table 1. Most studies were from different states in the United States (US), and used retrospective cohorts from administrative databases and various analyses with various levels of sophistocation.

Very few cost assessments were found for Canada: according to a systematic review (3), MRSA infection cost the Canadian health system between $\$ 54$ million and $\$ 110$ million (2005 CAD\$) (direct attributable health care cost per year) including infection, colonization and infrastructure. The average cost per patient for MRSA infection was estimated to be $\$ 12,216$ (range $\$ 6,878$ to $\$ 17,553$ ) (3). In a conference publication, Muller et al (4) showed that $\$ 10$ million in expenses were needed to control an MRSA outbreak in Toronto (Ontario) in 2006 to 2007, increasing hospital cost per patient by $35 \%$. In studies from the US, Filice et al (5) estimated adjusted mean cost of medical services for MRSA in patients with low Charlson's score ( 0 to 3 ) to be $\$ 51,252$ (2007 US\$) (95\% CI $\$ 46,041$ to $\$ 56,464)$ versus $\$ 30,158$ (95\% CI $\$ 27,092$ to $\$ 33,225)$ for methicillin-sensitive Staphylococcus aureus, and up to $\$ 84,436$ (95\% CI $\$ 79,843$ to $\$ 89,029$ ) versus $\$ 59,245$ (95\% CI $\$ 56,016$ to $\$ 62,473)$ for a high Charlson's score $(\geq 4)$. Shorr et al (6) did not find significant differences between MRSA and methicillinsensitive Staphylococcus aureus in a crude analysis, but estimates of cost were both high $(\$ 70,028$ versus $\$ 71,186)$.

Only one study assessed the cost of VRE; performed in Vancouver, British Columbia (7), it involved a large sample $(n=1292)$ and showed that this infection would increase mean costs by $62 \%$ to reach as high as $\$ 17,949$ per patient $(95 \%$ CI $\$ 13,949$ to $\$ 21,464)$. Using the Nationwide Inpatient Sample database, Nguyen et al (8) found that VRE infection would increase the adjusted average cost of inflammatory bowel disease by $127 \%$.

We did not find any studies evaluating the financial impact of ESBL-producing organisms in Canada. Only one US study investigating ESBL-producing organisms was found (9). This small, matchedcohort study $(\mathrm{n}=42)$ was performed in an 810 -bed community hospital (Hartford, Connecticut, USA). It compared patients infected with ESBL-producing Escherichia coli or Klebsiella species at a site other than the urinary tract with control patients infected with a non-ESBL-producing organism. The total mean infection-related costs were $\$ 41,353$ (2004 US\$) for cases of infections with ESBLproducing organisms and $\$ 24,902$ for controls $(\mathrm{P}=0.034)$. Infectionrelated $x L O S$ was prolonged in patients infected with ESBL compared with controls ( 21 days versus 11 days; $\mathrm{P}=0.006$ ); most of the difference in cost was directly related to the xLOS.

Another major HAI is Clostridium difficile infection (CDI). No recent national estimation of incidence is available in Canada and the latest estimation of cost, in 2008 US\$, was found in a systematic review (10): the mean total cost for a primary case of CDI would be $\$ 12,099$. According to the US NNIS incidence data, CDIs represent $30 \%$ of nationwide annual HAIs, with a cost of approximately $\$ 1.5$ billion (2). Lipp et al (11) estimated the annual cost of health care-associated CDI (2007 to 2008) extrapolated to all nonfederal US hospitals to be $\$ 792$ million, and a mean cost per case of $\$ 11,285$ ( $\$ 9,118$ to $\$ 13,574)$ (2). Three studies investigating CDI cost completed in different US states and a simulation model were retrieved and are shown in Table 1 . The results of the study by Campbell et al (12) highlight the higher total costs in patients exposed to concurrent antibiotics compared with elderly patients or those with renal impairment: $\$ 17,015$ (2010 US $\$$ ) (95\% CI $\$ 9,575$ to $\$ 24,456)$ were needed per case of CDI.

Several intervention studies have shown a positive association between antimicrobial stewardship and a decrease in the incidence of MRSA and CDI, either alone or as a component of a bundle (13-16). Fewer studies are available to support the same relationship with ESBLproducing organisms $(17-20)$, but it is reasonable to assume that decreasing overuse and misuse of antimicrobials should have a positive effect on most hospital antibiotic-resistant pathogens. Interventions associated with a decrease in antimicrobial consumption are inevitably linked with a decrease in antimicrobial costs as well, making these strategies even more interesting from the payers' and providers' perspectives. In addition, many different infection-control strategies have also been shown to decrease the incidence of HAIs, CDI and multidrug-resistant bacteria (21). These strategies have been evaluated alone or as part of multifaceted approaches, mainly on modifiable risk factors for HAI acquisition. The key strategies are hand hygiene, isolation of cases, education programs and compliance with good practice guidelines.

Nosocomial infections are associated with major burden of illness and use of health care resources. The massive expenditures of limited

${ }^{1}$ Department of Microbiology-Infectious Diseases, Université de Sherbrooke, Sherbrooke, Quebec; ${ }^{2}$ Departments of Medicine, Critical Care Medicine, Pathology and Laboratory Medicine and Community Health Sciences, University of Calgary, Calgary, Alberta; ${ }^{3}$ Department of Medicine, Royal Inland Hospital, Kamloops, British Columbia

Correspondence: Dr Louis Valiquette, Department of Microbiology and Infectious Diseases, Centre hospitalier universitaire de Sherbrooke, 3001, 12ème Avenue Nord, Sherbrooke, Quebec J1H 5N4. Telephone 819-346-1110 ext 12584, fax 819-829-3286,

e-mail louis.valiquette@usherbrooke.ca 
health care dollars required to manage these infections, as outlined in the present note, are neither new nor surprising. Considering an annual discount rate of $5 \%$ in health economic evaluations (22), the future trends are not likely to be decreasing soon. We believe that these data are needed to convince health care administrators to prioritize infection prevention and control resourcing according to the magnitude of this problem. In our personal experiences, funding of infection prevention and control efforts occurs at a small fraction of the cost likely incurred by nosocomial infections, while in some jurisdictions worldwide, use of funding penalties has been implemented in an attempt to motivate health care institutions to reduce nosocomial infection incidence. However, such an approach potentially results in minimizing funds to health care institutions with the greatest need for resources to control nosocomial infections. Perhaps an alternative would be to centrally and specifically fund infection prevention control programs at all health care institutions at the level that we are currently paying to deal with these infections. This would allow generous funding of programs that would stimulate enhanced and innovative preventive efforts. Effective institutions would inevitably have money 'left over' following successful prevention of infection that, in turn, could be used to benefit other areas within the hospital, or perhaps be even used as cash incentives to staff to enhance performance. Audits of hand-washing compliance of physicians and health care workers typically demonstrate dismal results despite repeated efforts to do so. Would physicians and health care workers improve these and other preventive efforts if we specifically paid them to do this?

TABLE 1

Cost of infections in published studies (PubMed, 2003-2013)

\begin{tabular}{|c|c|c|c|c|c|}
\hline Study & $\begin{array}{l}\text { Setting, } \\
\text { year of data }\end{array}$ & Methods* & $\begin{array}{l}\text { Perspective; } \\
\text { temporal } \\
\text { horizon; cost } \\
\text { adjustment }\end{array}$ & $\mathbf{n}$ & Main results \\
\hline \multicolumn{6}{|c|}{ Methicillin-resistant Staphylococcus aureus (MRSA) } \\
\hline $\begin{array}{l}\text { Labreche } \\
2013 \\
(23)\end{array}$ & $\begin{array}{l}\text { South Texas, USA } \\
13 \text { primary care } \\
\text { clinics } \\
\text { administrative } \\
\text { database, } \\
\text { 2009-2011 }\end{array}$ & $\begin{array}{l}\text { Prospective cohort of adults with SSTI; } \\
\text { 90-day follow-up } \\
\text { MRSA moderate or complicated vs mild or } \\
\text { uncomplicated }\end{array}$ & $\begin{array}{l}\text { Health } \\
\text { insurance } \\
\text { payer; } \\
\text { 32-month study } \\
\text { period; } \\
2011 \text { US } \$\end{array}$ & 265 & $\begin{array}{l}\text { Mean additional cost of treatment failure/case: } \$ 1,933 \text {; } \\
\$ 1,255 \text { in mild or uncomplicated, } \$ 2,093 \text { in moderate } \\
\text { or complicated }\end{array}$ \\
\hline $\begin{array}{l}\text { Filice } 2010 \\
\text { (5) }\end{array}$ & $\begin{array}{l}\text { Minneapolis, } \\
\text { Minnesota, USA } \\
\text { Veterans Affairs } \\
\text { database, } \\
\text { 2004-2006 }\end{array}$ & $\begin{array}{l}\text { Retrospective cohort, patients with } \\
\text { S aureus disease } \\
\text { MRSA vs MSSA } \\
\text { Semilogarithmic least-squares multivariate } \\
\text { model }\end{array}$ & $\begin{array}{l}\text { Hospital, } \\
\text { society; } 2007 \\
\text { US\$ }\end{array}$ & $\begin{array}{l}725 / \\
355: 390\end{array}$ & $\begin{array}{l}\text { Unadjusted median health care cost: } \$ 34,657 \\
\text { (range: } \$ 11,517-\$ 98,287) \\
\text { Overall median inpatient median cost: } \$ 26,274 \\
\text { (range } \$ 4,531-\$ 86,974) \text { vs } \$ 6,748 \text { for } M S S A(P<0.001) \\
\text { Overall median outpatient median cost: } \$ 4,322 \\
\text { (range } \$ 1,395-\$ 9,438) \text { vs } \$ 4,495(P=0.30)\end{array}$ \\
\hline $\begin{array}{l}\text { Shorr } \\
2010(6)\end{array}$ & $\begin{array}{l}\text { Detroit, Michigan, } \\
\text { USA } \\
\text { 903-bed tertiary care } \\
\text { center database, } \\
\text { 2005-2008 }\end{array}$ & $\begin{array}{l}\text { Retrospective cohort, inpatients with } \\
\text { pneumonia and blood or respiratory } \\
\text { culture of S aureus } \\
\text { MRSA vs MSSA }\end{array}$ & $\begin{array}{l}\text { Hospital; study } \\
\text { period; not } \\
\text { indicated }\end{array}$ & $\begin{array}{l}142 / \\
55: 87\end{array}$ & $\begin{array}{l}\text { Median total charges } \\
\$ 70,028 \text { vs } \$ 71,186 \text { MSSA ( } P=N S)\end{array}$ \\
\hline $\begin{array}{l}\text { Weigelt } \\
2010 \\
(24)\end{array}$ & $\begin{array}{l}\text { Marlborough, } \\
\text { Massachusetts, USA } \\
97 \text { acute care } \\
\text { hospitals clinical } \\
\text { research database, } \\
2003-2007\end{array}$ & $\begin{array}{l}\text { Retrospective cohort, postoperative } \\
\text { infection and positive surgical site } \\
\text { culture } \\
\text { MRSA vs other organisms } \\
\text { Multivariate logistic regression }\end{array}$ & $\begin{array}{l}\text { Hospital; not } \\
\text { indicated }\end{array}$ & 8302 & $\begin{array}{l}\text { Adjusted attributable cost vs other non-MRSA } \\
\text { monomicrobial infections: } \$ 1,157 \\
\text { (95\% CI } \$ 641-\$ 1,644) ; \mathrm{P}<0.0001 \\
\text { Median raw cost (mono and polymicrobial): } \$ 7,036 \\
\text { (IQR } \$ 4,024-\$ 11,989)\end{array}$ \\
\hline \multicolumn{6}{|c|}{ Vancomycin-resistant enterococci (VRE) } \\
\hline $\begin{array}{l}\text { Lloyd- } \\
\text { Smith } \\
2013(7)\end{array}$ & $\begin{array}{l}\text { Vancouver, British } \\
\text { Columbia } \\
\text { Urban hospital } \\
\text { database, } \\
\text { 2008-2009 }\end{array}$ & $\begin{array}{l}\text { Retrospective cohort, inpatients with VRE } \\
\text { colonization or infection; } \\
\text { VRE colonization or infection vs inpatients } \\
\text { without VRE } \\
\text { General linear model }\end{array}$ & $\begin{array}{l}\text { Hospital; not } \\
\text { indicated }\end{array}$ & $\begin{array}{l}1292 / \\
217: 1075\end{array}$ & $\begin{array}{l}\text { Mean total cost of care: } \$ 46,924 / \text { case vs } \$ 13,069 ; \\
\text { Absolute mean attributable cost/days: } \$ 17,949 \\
(95 \% \mathrm{Cl} \$ 13,949-\$ 21,464) ; \\
\text { Relative attributable cost } 61.9 \%(95 \% \mathrm{Cl} 42.3-84.3) \\
\text { greater than total hospital cost/patient without VRE }\end{array}$ \\
\hline $\begin{array}{l}\text { Butler } \\
2010 \\
(25)\end{array}$ & $\begin{array}{l}\text { Missouri, USA } \\
\text { Academic hospital } \\
\text { database, } \\
\text { 2002-2003 }\end{array}$ & $\begin{array}{l}\text { Retrospective cohort, nonsurgical patients } \\
\text { with first-episode enterococcal BSI; } \\
\text { VRE BSI and VSE BSI vs never BSI; } \\
\text { Standard GLS regression, propensity } \\
\text { score-weighted regression and propen- } \\
\text { sity score-matched pairs }\end{array}$ & $\begin{array}{l}\text { Hospital; } \\
\text { study period; } \\
2007 \text { US\$ }\end{array}$ & $\begin{array}{l}276 / \\
94: 182 \\
\text { controls } \\
20,150\end{array}$ & $\begin{array}{l}\text { Crude median hospital cost (IQR) for VRE: } \$ 42,106 \\
(\$ 16,310-\$ 93,870) \text {; VSE: } \$ 20,895(\$ 11,263- \\
\$ 41,879) \text {; controls: } \$ 8,192(\$ 5,615-\$ 13,495) \\
\text { Adjusted mean cost }(95 \% \mathrm{Cl}) \text { : } \\
\text { Using GLS: } \$ 4,479(\$ 3,500-\$ 5,732) \text { for VRE BSI, } \\
\$ 2,250(\$ 1,758-\$ 2,880) \text { for VSE BSI; } \\
\text { Using GLS + probability weighting: } \$ 4,036(\$ 3.0170- \\
\$ 5,140) \text { and } \$ 2,023(\$ 1,588-\$ 2,575) ; \\
\text { Using matched pairs: } \$ 9,949(\$ 1,579-\$ 24,693) \text { and } \\
\$ 5,282(\$ 2,042-\$ 8,043)\end{array}$ \\
\hline $\begin{array}{l}\text { Nguyen } \\
2011 \text { (8) }\end{array}$ & $\begin{array}{l}\text { Nationwide Inpatient } \\
\text { Sample database } \\
\text { of acute care } \\
\text { hospitals } \\
\text { 1998-2004 }\end{array}$ & $\begin{array}{l}\text { Retrospective cohort, IBD inpatients; } \\
\text { Discharge diagnosis ulcerative colitis or } \\
\text { Crohn's disease vs non-IBD GI disorder } \\
\chi^{2}, \text { multivariate adjustment }\end{array}$ & $\begin{array}{l}\text { Hospital; not } \\
\text { indicated } \\
2005 \text { US\$ }\end{array}$ & $\begin{array}{l}\text { 116,842: } \\
919,408\end{array}$ & $\begin{array}{l}\text { Crude total hospital charges } \$ 63,517 \text { vs } \$ 21,918 \text { for } \\
\text { no VRE; } P<0.001 \\
\text { Crude average charges/day: } \$ 3,272 \text { vs } \$ 3,124 \text { for no } \\
\text { VRE; } P=0.546 \text {; } \\
\text { Adjusted average total charges } 127 \% \text { higher with } \\
\text { VRE }(95 \% \mathrm{Cl} 85 \%-177 \%)\end{array}$ \\
\hline
\end{tabular}




\begin{tabular}{|c|c|c|c|c|c|}
\hline Study & $\begin{array}{l}\text { Setting, } \\
\text { year of data }\end{array}$ & Methods* & $\begin{array}{l}\text { Perspective; } \\
\text { temporal } \\
\text { horizon; cost } \\
\text { adjustment }\end{array}$ & $\mathbf{n}$ & Main results \\
\hline \multicolumn{6}{|c|}{ Clostridium difficile infection (CDI) } \\
\hline $\begin{array}{l}\text { Campbell } \\
2013 \\
(12)\end{array}$ & $\begin{array}{l}\text { Kansas City, } \\
\text { Missouri, } \\
74 \text { hospitals, } \\
\text { electronic health } \\
\text { record database } \\
2005-2011\end{array}$ & $\begin{array}{l}\text { Retrospective cohort, adult inpatients, five } \\
\text { high-risk patient groups: } \geq 65 \text { years of } \\
\text { age, renal disease, cancer, IBD, } \\
\text { concurrent antibiotic use } \\
\text { Propensity matching: HA-CDI vs non-CDI } \\
\text { Multivariate adjustment }\end{array}$ & $\begin{array}{l}\text { Hospital; } \\
\text { Study period } \\
2010 \text { US\$ }\end{array}$ & 4521 & $\begin{array}{l}\text { Difference in total costs HA-CDI vs non-CDI }(95 \% \mathrm{Cl}) \text { : } \\
\text { Age } \geq 65 \text { years: } \$ 6,906(\$ 3,942-\$ 9,871) \\
\text { Concurrent antibiotics : } \$ 17,015(\$ 9,575-\$ 24,456) \\
\text { Renal impairment : } \$ 4,604(\$ 1,025-\$ 8,182)\end{array}$ \\
\hline $\begin{array}{c}\text { Tabak } \\
2013 \\
(26)\end{array}$ & $\begin{array}{l}\text { Pennsylvania, six } \\
\text { hospitals, } \\
\text { administrative } \\
\text { database } \\
\text { 2007-2008 }\end{array}$ & $\begin{array}{l}\text { Retrospective cohort, adult inpatients with } \\
\text { primary HA-CDI } \\
\text { Propensity score matching: HA-CDI vs } \\
\text { non-CDI; Random-effects model }\end{array}$ & $\begin{array}{l}\text { Hospital; } \\
\text { Not indicated }\end{array}$ & 255 vs 765 & $\begin{array}{l}\text { Increase in attributable cost/year: } \$ 6,117 \\
(95 \% \mathrm{CI} \$ 1,659-\$ 10,574) \\
\text { Mean ( } \pm \text { SD) cost/case: } \$ 32,237 \pm 43,036 \\
\text { Median cost/case } \$ 20,804(\text { IQR } \$ 11,059-\$ 38,429)\end{array}$ \\
\hline $\begin{array}{l}\text { Lipp } 2012 \\
\quad(11)\end{array}$ & $\begin{array}{l}\text { New York State } \\
\text { All nonfederal acute } \\
\text { care hospitals } \\
\text { administrative } \\
\text { database } \\
\text { 2007-2008 }\end{array}$ & $\begin{array}{l}\text { Retrospective cohort of HA-CDI } \\
\text { General linear model }\end{array}$ & $\begin{array}{l}\text { Hospital and } \\
\text { state; } \\
\text { Study period } \\
\text { (two years); } \\
\text { Cost/charge } \\
\text { ratios; } \\
\text { Medicare } \\
\text { hospital cost } \\
\text { report }\end{array}$ & $4,853,800$ & $\begin{array}{l}\text { Average cost per infection: } \$ 29,000 \text {; } \\
\text { Annual cost in state: } \$ 55 \text { million }\end{array}$ \\
\hline $\begin{array}{l}\text { McGlone } \\
2011(27)\end{array}$ & $\begin{array}{l}\text { United States } \\
\text { hospitals } \\
\text { Computational } \\
\text { simulation model }\end{array}$ & $\begin{array}{l}\text { HA-CDI } \\
\geq 65 \text { years; mild or severe CDI; } \\
\text { Recurrence and progression to fulminant } \\
\text { colitis accounted for }\end{array}$ & $\begin{array}{l}\text { Hospital, third- } \\
\text { party payer } \\
\text { and societal; } \\
2010 \text { US\$ } \\
3 \% \text { discount/ } \\
\text { year }\end{array}$ & $\begin{array}{l}1000 / \\
\text { simulation }\end{array}$ & $\begin{array}{l}\text { Median cost }(95 \% \mathrm{Cl}) \text { of lost hospital bed days/first } \\
\text { occurrence and 6-day LOS: } \$ 7,511(\$ 6,868-\$ 8,210) \text {; } \\
\text { 14-day LOS: } \$ 9,539(\$ 8,342-\$ 10,994) \text {; } \\
\text { 3rd party direct costs: } \$ 8,237(\$ 7,563-\$ 9,014) \text {; } \\
\text { Direct and indirect costs/CDI and 6-day LOS: } \$ 14,726 \\
\quad(\$ 10,491-\$ 20,724)\end{array}$ \\
\hline
\end{tabular}

*Methods: Design, population, cases and comparison group, and analysis. BSI Bloodstream infection; GI Gastrointestinal; GLS Generalized least-squares; HA-CDI Hospital-acquired CDI; IBD Inflammatory bowel disease; IQR Interquartile range; LOS Length of stay; MSSA Methicillin-sensitive S aureus; SSTI Skin and soft tissues infection; vs Versus; VSE Vancomycin-sensitive enterococci

\section{REFERENCES}

1. Canadian Institute for Health Information. National Health Expenditure Trends, 1975 to 2013 - Executive Summary. Ottawa: CIHI, 2013.

2. Zimlichman E, Henderson D, Tamir O, et al. Health care-associated infections: A meta-analysis of costs and financial impact on the US health care system. JAMA Intern Med 2013;173:2039-46.

3. Goetghebeur M, Landry P A, Han D, Vicente C. Methicillin-resistant Staphylococcus aureus: A public health issue with economic consequences. Can J Infect Dis Med Microbiol 2007;18:27-34.

4. Muller MP, Jiang D, Nagulesapillai T, Melo M, Mamdani M. Impact of an MRSA outbreak on hospitals costs. Fifth Decennial International conference on health-care associated infections. Atlanta, Georgia, March 18 to 22, 2010.

5. Filice G A, Nyman JA, Lexau C, et al. Excess costs and utilization associated with methicillin resistance for patients with Staphylococcus aureus infection. Infect Control Hosp Epidemiol 2010;31:365-73.

6. Shorr AF, Haque N, Taneja C, et al. Clinical and economic outcomes for patients with health care-associated Staphylococcus aureus pneumonia. J Clin Microbiol 2010;48:3258-62.

7. Lloyd-Smith P, Younger J, Lloyd-Smith E, Green H, Leung V, Romney MG. Economic analysis of vancomycin-resistant enterococci at a Canadian hospital: Assessing attributable cost and length of stay. J Hosp Infect 2013;85:54-9.

8. Nguyen GC, Leung W, Weizman AV. Increased risk of vancomycinresistant enterococcus (VRE) infection among patients hospitalized for inflammatory bowel disease in the United States. Inflamm Bowel Dis 2011;17:1338-42.

9. Lee SY, Kotapati S, Kuti JL, Nightingale CH, Nicolau DP. Impact of extended-spectrum beta-lactamase-producing Escherichia coli and Klebsiella species on clinical outcomes and hospital costs: A matched cohort study. Infect Control Hosp Epidemiol 2006;27:1226-32.
10. Ghantoji SS, Sail K, Lairson DR, Dupont HL, Garey KW. Economic healthcare costs of Clostridium difficile infection: A systematic review. J Hosp Infect 2010;74:309-18.

11. Lipp MJ, Nero DC, Callahan MA. Impact of hospital-acquired Clostridium difficile. J Gastroenterol Hepatol 2012;27:1733-7.

12. Campbell R, Dean B, Nathanson B, Haidar T, Strauss M, Thomas S. Length of stay and hospital costs among high-risk patients with hospital-origin Clostridium difficile-associated diarrhea. J Med Econ 2013;16:440-8.

13. Kim YC, Kim MH, Song JE, et al. Trend of methicillin-resistant Staphylococcus aureus (MRSA) bacteremia in an institution with a high rate of MRSA after the reinforcement of antibiotic stewardship and hand hygiene. Am J Infect Control 2013;41:e39-43.

14. Jump RL, Olds DM, Seifi N, et al. Effective antimicrobial stewardship in a long-term care facility through an infectious disease consultation service: Keeping a LID on antibiotic use. Infect Control Hosp Epidemiol 2012;33:1185-92.

15. Nathwani D, Sneddon J, Patton A, Malcolm W. Antimicrobial stewardship in Scotland: Impact of a national programme. Antimicrob Resist Infect Control 2012;1:7.

16. Nowak M A, Nelson RE, Breidenbach JL, Thompson PA, Carson PJ. Clinical and economic outcomes of a prospective antimicrobial stewardship program. Am J Health Syst Pharm 2012;69:1500-8.

17. Knudsen J D, Andersen SE. A multidisciplinary intervention to reduce infections of ESBL- and AmpC-producing, Gram-negative bacteria at a university hospital. PLoS One 2014;9:e86457.

18. Dancer SJ, Kirkpatrick P, Corcoran DS, Christison F, Farmer D, Robertson C. Approaching zero: Temporal effects of a restrictive antibiotic policy on hospital-acquired Clostridium difficile, extendedspectrum beta-lactamase-producing coliforms and meticillin-resistant Staphylococcus aureus. Int J Antimicrob Agents 2013;41:137-42. 
19. Hanberger H, Arman D, Gill H, et al. Surveillance of microbial resistance in European Intensive Care Units: A first report from the Care-ICU programme for improved infection control. Intensive Care Med 2009;35:91-100.

20. Marchaim D, Chopra T, Bhargava A, et al. Recent exposure to antimicrobials and carbapenem-resistant Enterobacteriaceae: The role of antimicrobial stewardship. Infect Control Hosp Epidemiol 2012;33:817-30.

21. Davey P, Brown E, Charani E, et al. Interventions to improve antibiotic prescribing practices for hospital inpatients. Cochrane Database Syst Rev 2013;(4):CD003543.

22. Drummond MF, Sculpher MJ, Torrance GW, O'Brien BJ, Stoddart GL. Methods for the Economic Evaluation of Health Care Programmes, 3rd edn. Oxford Medical Publication. New York: Oxford University Press Inc, 2005.

23. Labreche MJ, Lee GC, Attridge RT, et al. Treatment failure and costs in patients with methicillin-resistant Staphylococcus aureus
(MRSA) skin and soft tissue infections: A South Texas Ambulatory Research Network (STARNet) study. J Am Board Fam Med 2013;26:508-17.

24. Weigelt JA, Lipsky BA, Tabak YP, Derby KG, Kim M, Gupta V. Surgical site infections: Causative pathogens and associated outcomes. Am J Infect Control 2010;38:112-20.

25. Butler AM, Olsen MA, Merz LR, et al. Attributable costs of enterococcal bloodstream infections in a nonsurgical hospital cohort. Infect Control Hosp Epidemiol 2010;31:28-35.

26. Tabak YP, Zilberberg MD, Johannes RS, Sun X, Mcdonald LC. Attributable burden of hospital-onset Clostridium difficile infection: A propensity score matching study. Infect Control Hosp Epidemiol 2013;34:588-96.

27. Mcglone SM, Bailey RR, Zimmer SM, et al. The economic burden of Clostridium difficile. Clin Microbiol Infect 2012;18:282-9. 


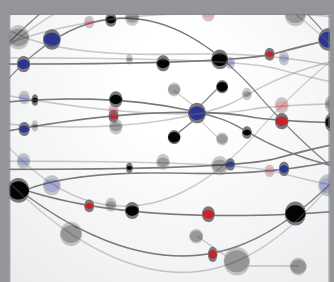

The Scientific World Journal
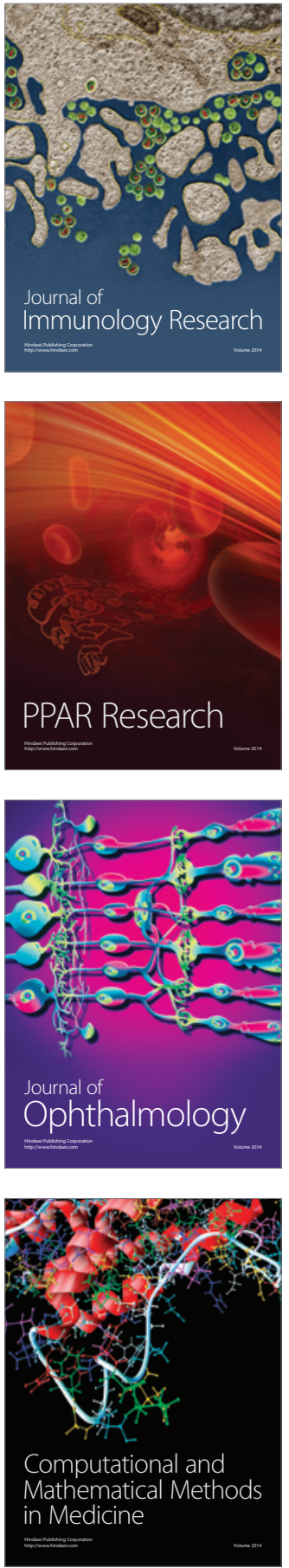

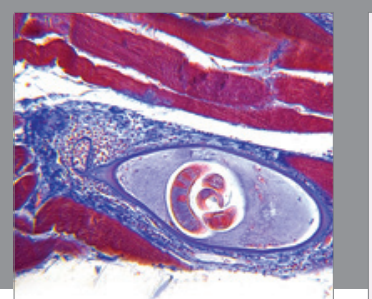

Gastroenterology Research and Practice

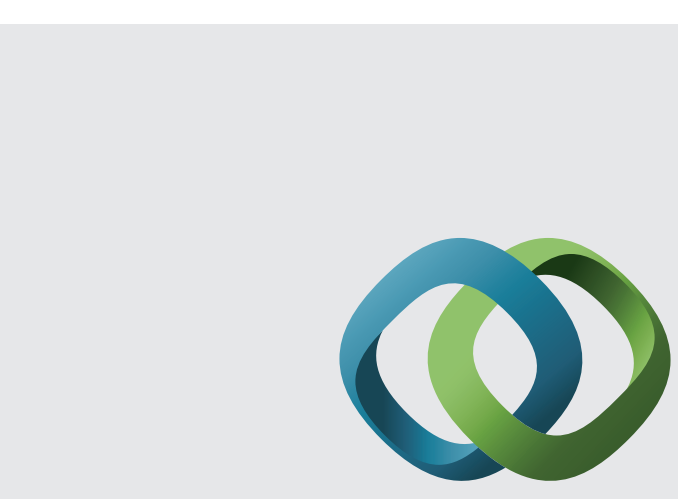

\section{Hindawi}

Submit your manuscripts at

http://www.hindawi.com
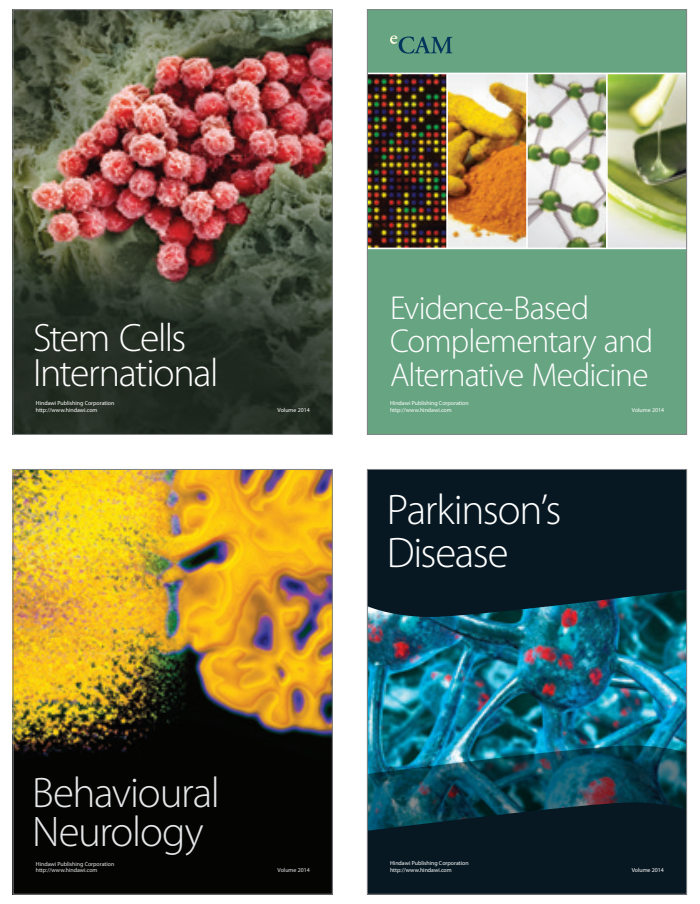
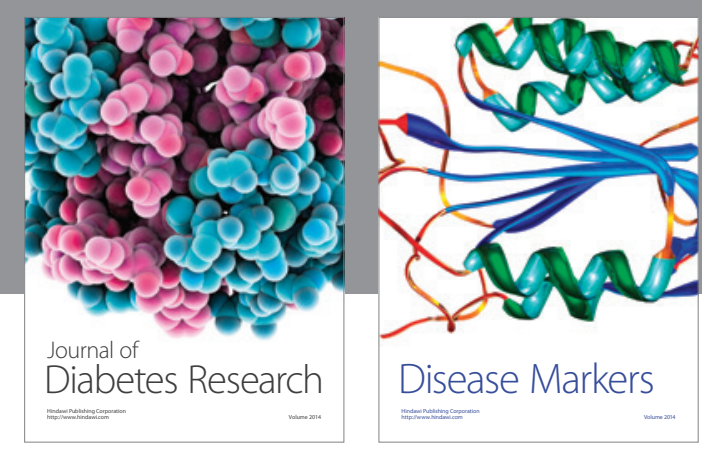

Disease Markers
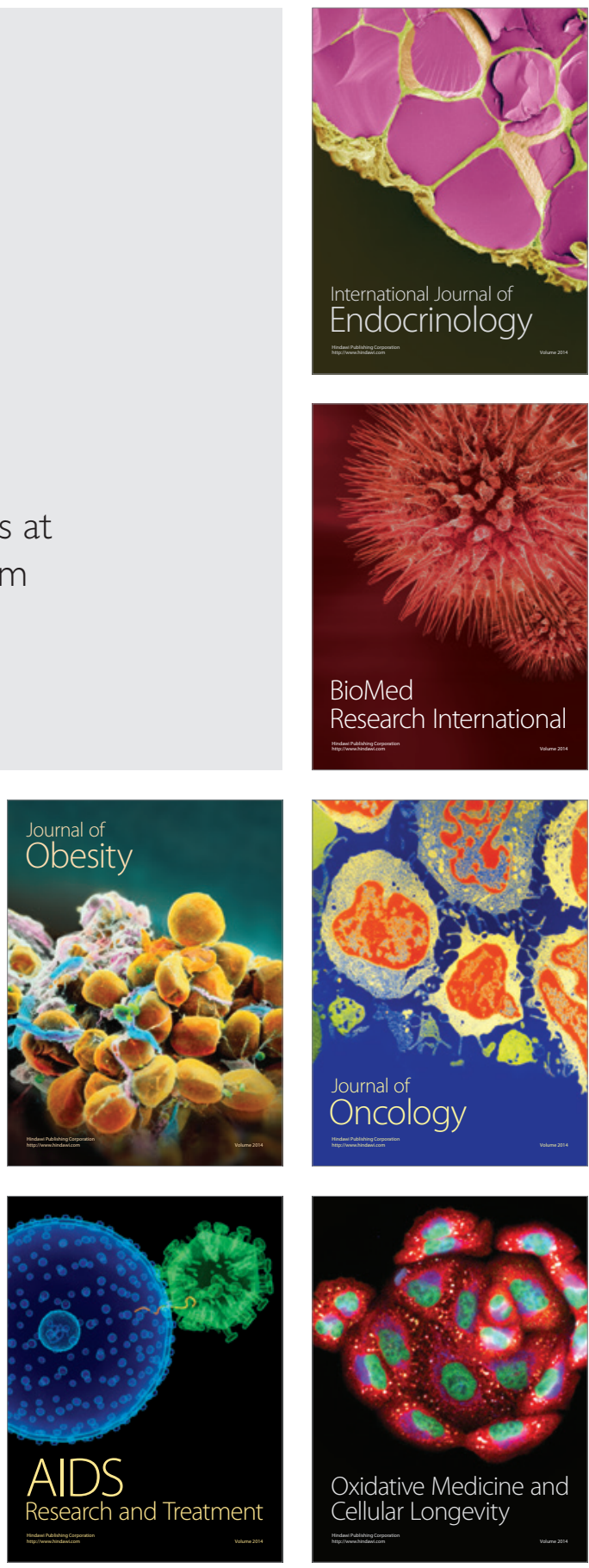